\title{
POTENCIAL, ECONOMIA DE ÁGUA E ADUBAÇÃO COM A APLICAÇÃO DE EFLUENTE DO TRATAMENTO PRELIMINAR DE ESGOTO DOMÉSTICO NA FERTIRRIGAÇÃO DE CAPIM-ELEFANTE
}

\author{
M. V. A. MARQUeS*, A. T. MATOS, A. P. M. PEREIRA, T. H. R. SILVÉRIO, D. L. A. PENIDO, M. T. M. COSTA, D. A. P. \\ SILVA \\ Universidade Federal de Minas Gerais - UFMG \\ marcus.ifnmg@yahoo.com.br*
}

Submetido 06/02/2017 - Aceito 03/04/2017

DOI: $10.15628 /$ holos.2017.5612

\begin{abstract}
RESUMO
A fertirrigação como alternativa de disposição final de esgoto doméstico é uma solução viável, tendo em vista o baixo custo de sua implantação, o aproveitamento dos nutrientes pelas plantas e a obtenção de melhorias na estruturação do solo. Neste trabalho, será avaliado o potencial do uso de efluente do tratamento preliminar de esgoto doméstico (EDP), a economia de água na irrigação e na adubação, considerando-se sua qualidade química para fertirrigação do capim-elefante (Pennisetum purpureum). Cada parcela experimental foi constituída por 4 linhas plantadas e 3 sulcos de aplicação de água/esgoto doméstico, sendo o espaçamento entre linhas de $0,6 \mathrm{~m}$ e o comprimento de $40 \mathrm{~m}$. Foram impostos dois tratamentos: T1 - aplicação de adubação química convencional e água potável, em irrigação; e T2 - aplicação, em fertirrigação, de EDP,
\end{abstract}

sendo a necessidade hídrica das plantas complementada com água da rede de abastecimento. A dose de aplicação do EDP foi estabelecida considerando-se suas características químicas e as necessidades nutricionais e de água do capim-elefante. Os resultados obtidos demonstraram que o EDP utilizado na fertirrigação do capim-elefante, considerando-se uma lâmina de aplicação para fornecimento de $300 \mathrm{~kg} \mathrm{ha}^{-1} \mathrm{ano}^{-1}$ de $\mathrm{Na}$, foi capaz de suprir $100 \%$ do $\mathrm{P}_{2} \mathrm{O}_{5}, 33 \%$ do $\mathrm{K}_{2} \mathrm{O}$ e $93 \%$ do $\mathrm{N}$ demandado na adubação do capim-elefante, gerando uma economia financeira de US\$ $445,00 \mathrm{ha}^{-1}$, no que se refere à adubação; de US\$ 624,00 ha ${ }^{-1}$, no que se refere ao custo de produção; e de US\$ $6,00 \mathrm{ha}^{-1}$, no que se refere ao custo de irrigação, proporcionando uma economia total de US\$ 630,00 ha $^{-1}$ e uma economia hídrica de $58 \%$ do total demandado.

PALAVRAS-CHAVE: Custo de produção, custo de irrigação, economia hídrica.

\section{POTENTIAL, WATER AND FERTILIZATION ECONOMY WITH APPLICATION OF PRELIMINARY TREATMENT EFFLUENT OF DOMESTIC SEWAGE IN FERTIGATION OF ELEPHANT GRASS}

\begin{abstract}
Fertigation as an alternative of final disposal of domestic sewage is a viable solution, considering the low cost of its implantation, the use of nutrients by the plants and the obtaining of improvements in the soil structure. In this work, the potential of the effluent of the preliminary domestic sewage treatment (PST), the water savings in irrigation and fertilization, will be evaluated, considering its chemical quality for elephant grass (Pennisetum purpureum) fertigation. Each experimental plot consisting of 4 planted lines and 3 water/domestic sewage application grooves, between $0.6 \mathrm{~m}$ and $40 \mathrm{~m}$ long lines. Two treatments were imposed: T1 application of conventional chemical fertilization and drinking water, in irrigation; and T2 - application in PST
\end{abstract}

fertigation, being the water requirement of the plants complemented with water from the supply network. The application rate of EDP was established considering its chemical characteristics and the nutritional and water needs of elephant grass. The results showed that the EDP used in elephant grass fertigation, considering an application sheet for to supply $300 \mathrm{~kg} \mathrm{ha}^{-1} \mathrm{ano}^{-1}$ of $\mathrm{Na}$, was able to supply $100 \% \mathrm{P}_{2} \mathrm{O}_{5}, 33 \% \mathrm{~K}_{2} \mathrm{O}$, and $93 \% \mathrm{~N}$ of elephant grass needs, generating a financial savings of US\$ $445.00 \mathrm{ha}^{-1}$ for fertilization; US\$ $624.00 \mathrm{ha}^{-1}$ of the production costs; and US\$ $6.00 \mathrm{ha}^{-1}$ of the irrigation costs, providing a total saving of US\$ $630.00 \mathrm{ha}^{-1}$ and a saving of $58 \%$ of the total water demanded.

KEYWORDS: Cost of production, cost of irrigation, water economy. 


\section{INTRODUÇÃO}

Com a escassez de água que atinge grande parte da população mundial, estratégias que diminuam a utilização de água de boa qualidade, ou seja, reduzam a dependência de captação das águas de corpos hídricos superficiais e subterrâneos, vêm sendo discutidas e estudadas. A magnitude desse problema se torna maior no meio rural, em razão do grande consumo de água na irrigação de culturas agrícolas.

A utilização de águas residuárias, tratadas ou não, na fertirrigação de culturas agrícolas tem sido há muito tempo pesquisada, pois, além de possibilitar o aporte de nutrientes essenciais ao desenvolvimento das plantas e matéria orgânica para melhorar a qualidade do solo, pode suprir parte da necessidade hídrica das plantas. Com isso, reduz-se a utilização de fontes de água de melhor qualidade, resguardando-se a utilização dessas para fins mais exigentes.

A utilização de água residuária, quando bem planejada, aplicando-se as doses recomendadas e nos períodos corretos, pode ser uma estratégia importante para proporcionar economia de água e insumos minerais em atividades agrícolas. As vantagens da reutilização da água residuária urbana está fundamentada na certeza de sua disponibilidade durante todo o ano, principalmente em cidades contempladas com rede de esgotamento sanitário, e na redução dos custos de seu tratamento (MOYO et al., 2015).

Para que esse processo seja vantajoso, do ponto de vista econômico e ambiental, algumas medidas devem ser levadas em consideração no momento de se definir a lâmina de água residuária a ser aplicada. As lâminas ou doses de aplicação não podem ser superiores às da capacidade de suporte do sistema solo-planta, para que não venha trazer prejuízos à cultura agrícola, ao solo e às águas subterrâneas. Além disso, aspectos sanitários devem ser observados, a fim de não causarem contaminação de alimentos e do meio ambiente.

Para manter uma alta produtividade na agricultura atual, necessita-se da adição de fertilizantes químicos, o que, geralmente, proporciona aumento considerável nos custos de produção agrícola. Nessa perspectiva, a utilização de água residuária poderá ser fonte de nutrientes para as plantas e, com isso, possibilitar redução na aplicação de adubos minerais em áreas de cultivo agrícola (KIHILA et al., 2014).

Em decorrência do já relatado problema atual de escassez de água, o aproveitamento agrícola de águas residuárias está deixando de ser uma prática aplicável apenas a regiões áridas para ser uma técnica corriqueira no mundo desenvolvido (HAMILTON et al., 2007). Há de se ressaltar, no entanto que, do ponto de vista financeiro e ambiental, o aproveitamento dos nutrientes no cultivo agrícola torna-se igualmente ou de maior importância que o do aproveitamento da própria água nos quais estão diluídos.

Em termos de conteúdo de nutrientes para plantas, o esgoto doméstico possui, em média, $45 \mathrm{mg} \mathrm{L}^{-1}$ de nitrogênio, $7 \mathrm{mg} \mathrm{L}^{-1}$ de fósforo, $158 \mathrm{mg} \mathrm{L}^{-1}$ de potássio, $53 \mathrm{mg} \mathrm{L}^{-1}$ de cálcio, 42 $\mathrm{mg} \mathrm{L}^{-1}$ de magnésio (THAPLIYAL et al., 2011; VON SPERLING, 2014). Isso demonstra o potencial de utilização dessa água residuária na fertilização de solos agrícolas, uma vez que esses nutrientes são essenciais para o desenvolvimento das plantas.

Os custos com adubação química vêm sendo um dos grandes entraves da agricultura convencional na atualidade, uma vez que para alcançar maiores produtividade, existe a necessidade do dispêndio de quantias significativas no processo de produção. Os valores gastos na compra de insumos variam de 30 - 60\% dos custos de produção (ALVES FILHO et al., 2003; NEUMANN et al., 2005) 
A irrigação por superfície, como a forma mais rudimentar de irrigação, tendo em vista o emprego o menor nível tecnológico, apresenta como vantagens o custo de implantação e manutenção, pois a distribuição de água na área se faz pela utilização do próprio solo, e não por tubulações. Segundo Bernardo et al. (2008), o valor de implantação desses sistemas pode chegar a $80 \%$ dos custos da aspersão convencional.

O objetivo com a condução desta pesquisa foi avaliar o potencial do uso de efluente do tratamento preliminar de esgoto doméstico, considerando-se sua qualidade química, no atendimento da demanda nutricional do capim-elefante (Pennisetum purpureum), além da economia de água de irrigação e de adubação química, quando utilizado na fertirrigação da referida forrageira.

\section{MATERIAL E MÉTODOS}

O experimento foi conduzido em área disponibilizada na Estação de Tratamento de Esgoto da COPASA (ETE - Onça), localizada junto ao Ribeirão do Onça, no município de Santa Luzia - MG, próximo a Belo Horizonte - MG, Brasil, nas coordenadas geográficas $19^{\circ} 49^{\prime} 20,6^{\prime \prime}$ Sul e $43^{\circ} 53^{\prime} 46,6^{\prime \prime}$ Oeste.

Antes do início do experimento, foi feita a caracterização física, química e físico-hídrica de amostras de solo coletadas nas profundidades de 0-20, 20-40 e 40-60 cm, no local onde foi instalado o experimento (TABELA 1). As análises químicas do solo consistiram em medição do potencial hidrogeniônico $(\mathrm{pH})$ e quantificação dos teores de matéria orgânica $(\mathrm{MO})$ e nitrogênio total (NT); trocáveis ou disponíveis de fósforo $\left(\mathrm{P}_{\text {disp }}\right)$, potássio $\left(\mathrm{K}_{\text {disp }}\right)$, sódio $\left(\mathrm{Na}_{\text {disp }}\right)$, cálcio $\left(\mathrm{Ca}_{\text {disp }}\right)$, magnésio $\left(\mathrm{Mg}_{\text {disp }}\right)$, Zinco $\left(\mathrm{Zn}_{\text {disp }}\right)$, Cobre $\left(\mathrm{Cu}_{\text {disp }}\right)$ e alumínio $\left(\mathrm{Al}_{\text {troc }}\right)$; além da capacidade de troca de cátions efetiva $\left(\mathrm{CTC}_{\mathrm{ef}}\right)$ e potencial $\left(\mathrm{CTC}_{\text {pot }}\right)$, acidez potencial do solo, porcentagem de saturação por bases e razão de adsorção de sódio (RAS), utilizando-se os métodos descritos em Embrapa (1999) e Matos (2012). Essas análises foram realizadas no Laboratório de Rotina do Departamento de Solos - UFV.

As análises físicas contemplaram a análise de massa específica global do solo $\left(\rho_{s}\right)$, análise granulométrica (argila, silte e areia), utilizando-se os métodos descritos em Embrapa (1999) e Matos (2012), cujas análises foram realizadas no Laboratório de Hidráulica do Departamento de Engenharia Agrícola - UFV, além da condutividade elétrica da suspensão (relação solo:água de $1: 2,5)$ do solo, realizadas no Laboratório de Rotina do Departamento de Solos - UFV.

A caracterização físico-hídrica do solo consistiu na quantificação do teor de água na capacidade de campo $\left(U_{c c}\right)$, quantificada após ser submetida às tensões de 0,1 e 0,3 bar, e no ponto de murcha permanente $\left(U_{\mathrm{pmp}}\right)$, quantificada após ser submetida à tensão de 15 bar, utilizando-se os métodos descritos em Embrapa (1999). Os valores de $U_{c c}\left(15,0\right.$ bar) e $U_{p m p}(0,1$ bar) foram utilizados como base para cálculo da lâmina de aplicação de água no solo (BERNARDO et al., 2008) como complemento ou, quando for o caso, para atendimento completo das necessidades hídricas das plantas. Essas análises foram realizadas no Laboratório de Hidráulica do Departamento de Engenharia Agrícola - UFV. 


\begin{tabular}{|c|c|c|c|c|c|c|c|c|c|c|c|}
\hline \multirow{2}{*}{$\begin{array}{l}\text { Prof } \\
(\mathrm{cm})\end{array}$} & pH & CE & MO & $\mathbf{N}_{\text {Total }}$ & $\mathbf{P}_{\text {Disp }}$ & $K_{\text {Disp }}$ & $\mathrm{Na}_{\text {Disp }}$ & $\mathrm{Zn}_{\text {Disp }}$ & $\mathrm{Cu}_{\text {Disp }}$ & Ca Disp & Mg \\
\hline & & $\mu \mathrm{S} \mathrm{cm}^{-1}$ & $\mathrm{~g} \mathrm{~kg}^{-1}$ & \multicolumn{8}{|c|}{ 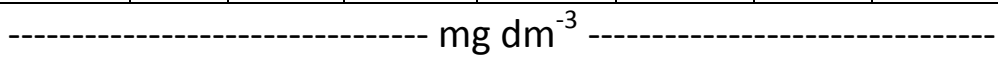 } \\
\hline $0-20$ & 7 & 93 & 6,34 & 0,48 & 2,6 & 54 & 48 & 21 & 30 & 1575 & 253 \\
\hline $20-40$ & 7,3 & 106 & 3,81 & 0,36 & 4 & 63 & 190 & 25 & 19 & 1872 & 252 \\
\hline $40-60$ & 7,4 & 92 & 3,81 & 0,36 & 5,7 & 67 & 41 & 21 & 17 & 1979 & 245 \\
\hline \multirow{2}{*}{$\begin{array}{l}\text { Prof } \\
(\mathrm{cm})\end{array}$} & $\mathrm{Al}_{\text {troc }}$ & $\mathrm{H}+\mathrm{Al}$ & $\mathrm{CTC}_{\mathrm{Ef}}$ & CTC $_{\text {Pot }}$ & V & m & $\rho_{s}$ & $\mathbf{U}$ & areia & silte & argila \\
\hline & \multicolumn{4}{|c|}{--------- $\mathrm{mmol}_{\mathrm{c}} \mathrm{dm}^{-3}$} & \multicolumn{2}{|c|}{----- \% ----- } & $\mathrm{g} \mathrm{cm}^{-3}$ & $\mathrm{~kg} \mathrm{~kg}^{-1}$ & \multicolumn{3}{|c|}{ 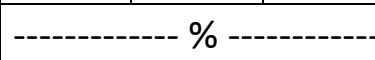 } \\
\hline $0-20$ & 1 & 7,5 & 104 & 111 & 93 & 1 & 1,26 & $0,187^{1}$ & 46,1 & 37 & 16,9 \\
\hline $20-40$ & 1 & 3 & 147 & 149 & 98 & 1 & - & $0,167^{2}$ & - & - & - \\
\hline $40-60$ & 1 & 3 & 124 & 126 & 98 & 1 & - & $0,075^{3}$ & - & - & - \\
\hline
\end{tabular}

Tabela 1: Resultados das análises químicas, físicas e físico-hídricas em amostras do solo da área experimental, coletados nas camadas de 0-20, 20-40 e 40-60 cm.

Em que: CE - condutividade elétrica medida na suspensão solo:água 1:2,5; H+Al - acidez potencial; $V$ - saturação por bases; $m$ - saturação por alumínio; $\rho_{\mathrm{s}}$ - massa específica global do solo; ${ }^{1,2,3}$ quantificada sob tensão de $0,1,0,3$ e 15 bar apenas para camada de 0-20 cm, respectivamente.

A fertirrigação com o efluente do tratamento preliminar do esgoto doméstico (EDP) na área de cultivo do capim-elefante foi efetuada por meio do método superficial, mais precisamente, sulcos. Para isso, o terreno foi previamente sistematizado, ou seja, com base em levantamento topográfico do local, teve sua superfície aplainada (BERNARDO et al., 2008). Os sulcos foram construídos após a sistematização da área com formato de trapezoidal, tendo profundidade de $0,20 \mathrm{~m}$, largura de $0,30 \mathrm{~m}$ e espaçamento de 0,60 $\mathrm{m}$. Os sulcos foram construídos sem declividade de fundo, ou seja, em nível.

Seguindo-se as instruções estabelecidas em Embrapa (2015), o plantio de capim-elefante foi efetuado por meio de mudas, optando-se por efetuar o plantio no espaçamento de 0,6 $\mathrm{m}$ entre linhas. Desta forma, foi construído um sulco entre cada linha de cultivo.

Cada parcela experimental foi constituída por três sulcos fechados e quatro linhas de cultivo, sendo os sulcos e as plantas laterais considerados bordaduras. Os sulcos foram construídos com $40 \mathrm{~m}$ de extensão, o que proporcionou uma área de cultivo total de $72 \mathrm{~m}^{2}$ por parcela experimental.

A adubação do solo das parcelas que não recebeu EDP foi feita via fertilização convencional, enquanto a fertirrigada não recebeu adubação química. Para adubação química convencional $(A Q C)$ do capim nas parcelas que não receberam $E D P$, receberam a dose equivalente a 6 meses de cultivo, foram aplicadas doses de $80 \mathrm{~kg} \mathrm{ha}^{-1}$ de $\mathrm{P}_{2} \mathrm{O}_{5}, 300 \mathrm{~kg} \mathrm{ha}^{-1}$ de N e de $\mathrm{K}_{2} \mathrm{O}$, utilizando-se os fertilizantes comerciais superfosfato simples (SFS), ureia (U) e cloreto de potássio $(\mathrm{KCl})$, respectivamente. A adubação nitrogenada e potássica foi aplicada de forma parcelada, em doses de $50 \mathrm{~kg} \mathrm{ha}^{-1}$ a cada corte, de acordo com a recomendação da Comissão de Fertilidade do Solo do Estado de Minas Gerais (RIBEIRO et al., 1999). O controle de plantas daninhas nas parcelas experimentais foi feito manualmente, com o auxílio de enxada.

A água residuária utilizada no experimento foi constituída pelo esgoto bruto, submetido apenas a um gradeamento (EDP), cuja caracterização foi feita, em termos de seus atributos físicos, químicos e bioquímicos apresentada na Tabela 2. As análises químicas do EDP contemplaram as medições do potencial hidrogeniônico $(\mathrm{pH})$, demanda química de oxigênio (DQO), demanda bioquímica de oxigênio (DBO), além das concentrações de nitrogênio total 
Kjeldahl (NTK), fósforo (P), potássio (K), sódio ( $\mathrm{Na})$, cálcio $(\mathrm{Ca})$, magnésio $(\mathrm{Mg})$, alumínio (Al), manganês ( $\mathrm{Mn})$, cobre $(\mathrm{Cu})$, zinco $(\mathrm{Zn})$ e ferro $(\mathrm{Fe})$.

As análises físicas do esgoto consistiram na quantificação dos sólidos totais (ST), sólidos fixos totais (SFT) e sólidos voláteis totais (SVT), além da condutividade elétrica (CE) na suspensão preparada com água.

Determinaram-se as concentrações de $\mathrm{Ca}, \mathrm{Mg}, \mathrm{Al}, \mathrm{Mn}, \mathrm{Cu}, \mathrm{Zn}$ e $\mathrm{Fe}$, por espectrofotometria de absorção atômica; $\mathrm{N}$, pelo método titulométrico; $\mathrm{Na}$ e $\mathrm{K}$, por cromatografia iônica; o $\mathrm{P}$ e DQO, pelo método colorimétrico; DBO, pelo método do oximétrico; ST, SFT e SVT, pelo método gravimétrico.

Os métodos utilizados nas análises estão apresentados Standard Methods for the Examination for Water and Wastewater (APHA, 2005) e em Matos (2015). Todas as análises foram realizadas no Laboratório de Físico-Química do Departamento de Engenharia Sanitária e Ambiental - UFMG e no Laboratório de Análises Químicas do Departamento de Engenharia Química - UFMG.

\begin{tabular}{|c|c|c|c|c|c|c|c|c|c|}
\hline & \multirow[b]{2}{*}{$\mathrm{pH}$} & \multirow{2}{*}{$\frac{\mathrm{CE}}{\mu \mathrm{S} \mathrm{cm}^{-1}}$} & ST & SFT & SVT & DBO & DQO & NTK & $\mathbf{P}$ \\
\hline & & & \multicolumn{7}{|c|}{ 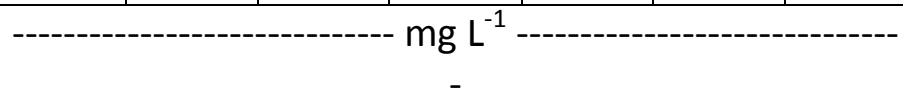 } \\
\hline Média & 7,7 & 1523 & 998 & 544 & 454 & 433 & 760 & 136,7 & 19,9 \\
\hline \multirow[t]{3}{*}{ Mediana } & 7,7 & 1543 & 992 & 542 & 453 & 435 & 718 & 128,6 & 19,2 \\
\hline & $\mathrm{K}$ & $\mathrm{Na}$ & Al & $M n$ & $\mathrm{Fe}$ & $\mathrm{Zn}$ & $\mathrm{Ca}$ & $\mathrm{Mg}$ & $\mathrm{Cu}$ \\
\hline & \multicolumn{9}{|c|}{ 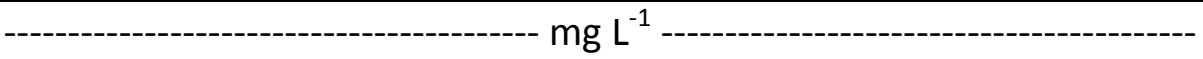 } \\
\hline Média & 40,6 & 79,5 & 4,2 & 1,10 & 3,3 & 0,35 & 21,3 & 6,4 & $<0,05$ \\
\hline Mediana & 43,0 & 83,7 & 3,0 & 0,14 & 3,5 & 0,28 & 20,9 & 5,9 & $<0,05$ \\
\hline
\end{tabular}

Tabela 2: Características médias do esgoto doméstico efluente do tratamento preliminar.

Para atendimento dos objetivos deste experimento, os tratamentos foram: T1 - parcelas experimentais receptoras de adubação química convencional e irrigação com água da rede de abastecimento e T2 - parcelas experimentais receptoras de EDP, via fertirrigação, sendo as necessidades hídricas das plantas completadas com água da mesma rede.

Com base na caracterização apresentada na Tabela 2, calculou-se a lâmina a ser aplicada na cultura do capim-elefante, a qual ficou estabelecida pela aplicação de $300 \mathrm{~kg} \mathrm{ha}^{-1} \mathrm{ano}^{-1} \mathrm{de} \mathrm{Na}$, via EDP, tal como sugerido por Matos (2014), para que esse elemento químico não seja aplicado em excesso no solo.

Para eliminar o fator disponibilidade de água entre os tratamentos que receberam EDP, a lâmina aplicada de EDP está sendo complementada com água da rede de abastecimento. $\mathrm{O}$ manejo da irrigação, tanto da parcela receptora de água da rede de abastecimento como na complementação do fornecimento de água na parcela receptora de EDP, foram estabelecidos com base na evapotranspiração da cultura (ETc). A ETc foi calculada conforme apresentado por Doorenbos e Pruitt (1977), que utiliza a evapotranspiração de referência (ETo), estimada de acordo com o método de Thornthwaite (1948), disponibilizado pelo INMET (Instituto Nacional de Meteorologia); e utilizando um coeficiente de cultura (Kc) que leva em consideração a cultura instalada e seus estágios de desenvolvimento (DOORENBONS; KASSAM, 1994). A temperatura 
média ( $(-\mathrm{C})$, a umidade relativa do ar média (\%), a precipitação acumulada $(\mathrm{mm})$ e a irrigação total necessária por semana $(\mathrm{mm})$ estão apresentadas na Tabela 3.

\begin{tabular}{l|c|c|c|c}
\hline Mês & $\mathbf{T}_{\text {média }}\left({ }^{\mathbf{C}} \mathbf{C}\right)$ & $\mathbf{U R}_{\text {média }}(\mathbf{\%})$ & $\mathbf{P}(\mathbf{m m})$ & $\mathbf{I T N}_{\text {semanal }}(\mathbf{m m})$ \\
\hline junho & 18 & 59 & 0 & 29 \\
\hline julho & 20 & 53 & 0 & 28 \\
\hline agosto & 21 & 49 & 9 & 33 \\
\hline setembro & 23 & 49 & 56 & 42 \\
\hline outubro & 24 & 53 & 31 & 48 \\
\hline novembro & 22 & 63 & 248 & 48 \\
\hline dezembro & 23 & 63 & 304 & 52 \\
\hline
\end{tabular}

Tabela 3: Temperatura média mensal, umidade relativa média mensal, precipitação acumulada mensal e irrigação total necessária em cada semana de cultivo do capim-elefante.

Como forma operacionalmente mais adequada do sistema, optou-se por efetuar a aplicação de água da rede de abastecimento na área considerando-se um turno de rega de 7 dias, ou seja, a irrigação e a fertirrigação têm sido aplicadas semanalmente. A aplicação de EDP foi realizada por 24 semanas ( 6 meses), período compreendido entre o plantio e o segundo corte da forrageira, iniciado em junho (inverno) e terminado em dezembro (verão).

Para avaliação dos ganhos financeiros com a aplicação da fertirrigação com o EDP no cultivo do capim-elefante, calcularam-se as doses aportadas ao solo (Equação 1) dos principais nutrientes necessários para o desenvolvimento do capim-elefante ( $N, P$ e $K$ ), que foram convertidos para $\mathrm{N}, \mathrm{P}_{2} \mathrm{O}_{5}$ e $\mathrm{K}_{2} \mathrm{O}$, respectivamente, para facilitar a comparação com valores apresentados na literatura. Estes resultados foram comparados com as recomendações de doses da AQC.

$\mathrm{Df}=\left(\sum_{1}^{\mathrm{n}} \frac{\mathrm{C} * \mathrm{~V}}{\mathrm{~A}}\right) *\left(\frac{\mathrm{MMm}}{\mathrm{MMeq}}\right)$

Em que: Df - dose aportada do elemento químico ao solo, via fertirrigação ( $\left.\mathrm{kg} \mathrm{ha}{ }^{-1}\right)$; $\mathrm{C}$ concentração do nutriente em questão $\left(\mathrm{kg} \mathrm{m}^{-3}\right) ; \mathrm{V}$ - volume aplicado $\left(\mathrm{m}^{3}\right) ; \mathrm{A}$ - área receptora (ha); $\mathrm{n}$ - semanas de aplicação; MMm - massa molar da molécula; e MMeq - massa molar do elemento químico.

A economia de água no período de cultivo foi calculada em percentagem, como sendo a parcela do volume de irrigação total necessária para o desenvolvimento da cultura, subtraído da precipitação em todo período amostrado, que foi atendido pela aplicação do EDP. A quantidade de água economizada foi convertida para valores em dólares (US\$), de acordo com a tarifa cobrada pelo Comitê de Bacia Hidrográfica do Rio das Velhas (CBH -Velhas, DN no 3/2009), para captação de águas superficiais.

O cálculo do custo para cada adubo químico (N, P e K) está apresentado na Equação 2 e a economia de adubo químico com a utilização da fertirrigação utilizando-se o EDP, na Equação 3. O custo de adubação foi baseado em valores levantados por pesquisa no comércio de atacado, 
todos os valores utilizados neste trabalho, foram convertidos para dólar e reajustados para janeiro de 2017.

$\mathrm{Caq}=\frac{\mathrm{Vaq}^{*} \mathrm{Daq}}{\mathrm{CAQC}}$

$\mathrm{Ef}=\frac{\mathrm{Caq} * \mathrm{Df}}{\mathrm{Daq}}$

Em que: Caq - custo da adubação química de um hectare para cultivo do capim-elefante (US\$ ha $\left.{ }^{1}\right)$; Vaq - valor financeiro do adubo químico, por unidade de massa (US\$ $\mathrm{kg}^{-1}$ ); Daq - dose do nutriente utilizada na área de cultivo $\left(\mathrm{kg} \mathrm{ha}^{-1}\right)$; $C \mathrm{AQC}$ - constituição centesimal do adubo químico (adimensional); e Ef - economia na adubação, por hectare, em razão da fertirrigação com o EDP (US\$ ha ${ }^{-1}$ ).

Foram estimados os custo de produção em função do custo com arrendamento da área, fertilizantes, mecanização e mão-de-obra, com base em referenciais obtidos em Alves Filho et al. (2003), Neumann et al. (2005) e Bernardo et al. (2008). Também foi estimado o custo de irrigação, sendo para isso, utilizada a Equação 4, adaptada da proposta de Soares et al. (2002).

$\mathrm{Cl}=\mathrm{Cim}+\mathrm{Capl}+\mathrm{Cm}+(\mathrm{Cag} * \mathrm{ITN})$

Em que: $\mathrm{Cl}$ - custo da irrigação (US\$ ha- ${ }^{-1}$ ); Cim - custo de implantação (US\$ ha ${ }^{-1}$ ); Capli - custo de aplicação (US\$ ha ${ }^{-1}$ ); Cm - custo de manutenção (US\$ ha ${ }^{-1}$ ); Cag - custo da água (US\$ $\mathrm{mm}^{-1} \mathrm{ha}^{-1}$ ); e ITN - irrigação total necessária de T1 e T2 (mm).

Para o Cim, foi considerado que a sistematização do solo foi à mínima possível e utilizaria o mesmo sistema para fertirrigação. O Capli foi considerado a mão-de-obra utilizada para aplicação da lâmina requerida durante o ciclo estudado. $0 \mathrm{Cm}$ foi considerado o equivalente a $3 \%$ do Cim, como sugerido por Leitch (1983) apud Soares et al. (2003). O Cag foi calculado de acordo com a tarifa cobrada pelo Comitê de Bacia Hidrográfica do Rio das Velhas (CBH -Velhas, DN no 3/2009).

\section{RESULTADOS E DISCUSSÕES}

Os resultados apresentados a seguir, fazem referência a dose recomendada de $\mathrm{Na}, 300 \mathrm{~kg}$ $\mathrm{ha}^{-1} \mathrm{ano}^{-1}$, o que, em 24 semanas, seria $163 \mathrm{~kg} \mathrm{ha}^{-1}$. Analisando-se os resultados apresentados na Tabela 2, verifica-se que o elemento químico referencial $\mathrm{Na}$ apresentou concentração média de $82,0 \mathrm{mg} \mathrm{L}^{-1}$ no EDP, o que proporcionou a aplicação de uma dose de $163 \mathrm{~kg} \mathrm{ha}^{-1}$ de Na no solo, em igual período de tempo (Tabela 4). Valores maiores de concentração de $\mathrm{Na}\left(121 \mathrm{mg} \mathrm{L}^{-1}\right.$ ) foram encontrados por Leal et al. (2009), em efluente oriundo de um sistema de tratamento de esgoto doméstico, composto por lagoas anaeróbias seguido de lagoas facultativas. Os autores, no intento de suprir $100 \%$ da demanda hídrica da cana-de-açúcar, aplicaram uma dose de $3.056 \mathrm{~kg}$ $\mathrm{ha}^{-1}$ de $\mathrm{Na}$, valor incompatível para que a prática da fertirrigação seja conduzida com mínimo impacto para o solo e/ou águas subterrâneas.

As concentrações de $\mathrm{N}$ e $\mathrm{P}$ no EDP foram, em média, de 142 e 20,8 $\mathrm{mg} \mathrm{L}^{-1}$ (Tabela 4), respectivamente, valores acima dos citados por Von Sperling (2014) para esgoto doméstico bruto. Sabe-se que esses valores são reduzidos consideravelmente em esgotos tratados, tendo 
em vista que parte importante dos nutrientes em solução são incorporados à biomassa do sistema e, no caso específico do $\mathrm{N}$, sofre processos de desnitrificação.

Segundo Thapliyal et al. (2011), em sistemas alagados construídos (wetlands construídos), a remoção de $N$ pode chegar a $40 \%$ e de $\mathrm{P}$ a $30 \%$. Kihila et al. (2014) analisando um sistema de tratamento de esgoto para fertirrigação, demonstraram que concentração de $\mathrm{N}$ que passa por lagoas facultativas e de maturação, diminui de 9,19 para $2,35 \mathrm{mg} \mathrm{L}^{-1}$ e quando submetido a um sistema alagado construído, posteriormente, ocorre ainda cerca de $25 \%$ de redução nessa concentração. Neste sentido, quando o esgoto doméstico é tratado, perde-se sua qualidade para uso agrícola, pois ocorre diminuição na quantidade de nutrientes essenciais $(N$ e $P$, principalmente) fornecidos às plantas.

\begin{tabular}{l|c|c|c|c}
\hline Variável & Na & NTK & $\mathbf{P}$ & K \\
\hline Concentração média $\left(\mathrm{mg} \mathrm{L}^{-1}\right)$ & 82,0 & 142 & 20,8 & 42,0 \\
\hline Dose aplicada $\left(\mathrm{kg} \mathrm{ha}^{-1}\right)$ & 163 & 280 & 41,5 & 83,0 \\
\hline
\end{tabular}

Tabela 4: Dose de Na, NTK, P e K, após 24 semanas de aplicação do EDP no solo.

A dose de $\mathrm{N}$ aplicada via EDP não superou $300 \mathrm{~kg} \mathrm{ha}^{-1}$ (Tabela 4), valor correspondente ao das necessidades das plantas, para 6 cortes, quantidade adotada para os tratamentos controle com adubação convencional, segundo recomendação da Comissão de Fertilidade do Solo do Estado de Minas Gerais Ribeiro et al. (1999). A concentração de P no EDP foi maior que a reportada por Fonseca et al. (2005) em esgoto doméstico tratado em lagoas anaeróbias, facultativas e de estabilização, que foi de $8,4 \mathrm{mg} \mathrm{L}^{-1}$ (TABELA 2).

A concentração de $\mathrm{K}$ no EDP (TABELA 2) foi maior do que a encontrada por Abdel-Aziz et al. (2015) em Gyza no Egito, em esgoto doméstico submetido a tratamento secundário, que foi de $22 \mathrm{mg} \mathrm{L}^{-1}$, e menor que a reportada por Thapliyal et al. (2011), que obtiveram, em Delhi, na Índia, $158 \mathrm{mg} \mathrm{L}^{-1}$, em esgoto doméstico bruto.

Na Figura 1 estão apresentados os gráficos box-whisker, nos quais pode ser observado que a concentração de P no EDP aplicado apresentou menor amplitude entre quartis, ou seja, menor coeficiente de variação, ao passo que a de NTK apresentou a maior variação, fato esperado pela sua grande instabilidade, em termos de forma iônica, e perdas que esse elemento químico apresenta no sistema.
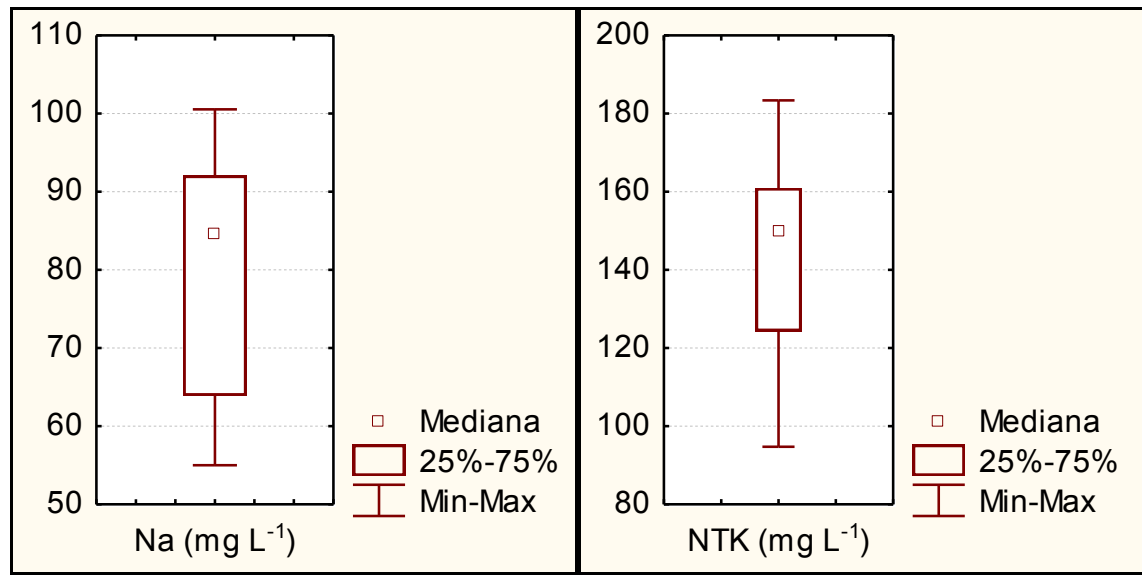


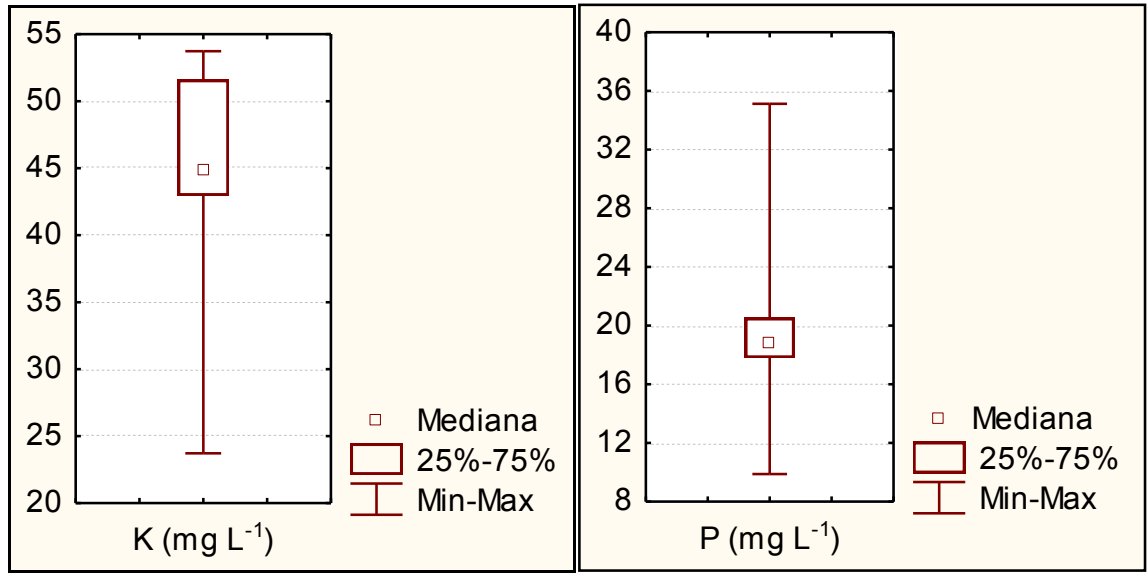

Figura 1: Gráficos box-whisker apresentando as medianas, amplitude entre quartis, mínimos e máximos, das concentrações de $\mathrm{Na}$, NTK, P e K no EDP.

A irrigação total necessária (ITN) na área, considerando-se as 24 semanas de cultivo foi de $370 \mathrm{~mm}$, enquanto a lâmina aplicada de EDP, considerando-se a utilização de $300 \mathrm{~kg} \mathrm{ha}^{-1} \mathrm{de} \mathrm{Na}$, foi de $214 \mathrm{~mm}$. Com base nisso, pode-se calcular que o uso do EDP representou uma economia de $58 \%$ na água que seria utilizada numa irrigação convencional do capim-elefante, o que de acordo com a tarifa cobrada no estado de Minas Gerais pelo CBH - Velhas, gerou uma economia de US\$ 6,00 ha ${ }^{-1}$ em 24 semanas. Cabe ressaltar que este valor de economia seria facilmente superado em esgotos domésticos, caso as concentrações de Na fossem menores, o que acontece em ETE que recebem o esgoto por meio de adutoras abertas, pois aumenta-se o fator de diluição.

$\mathrm{Na}$ tabela 5 estão apresentados os resultados relativos aos gastos com AQC, necessários para se efetuar 6 cortes na forrageira, a partir do plantio, tendo sido o custo total com AQC de US\$ 570,00 ha ${ }^{-1}$. Este valor foi superior ao encontrado por Neumann et al. (2005), que foi de cerca de US\$153,50 $\mathrm{ha}^{-1}$, utilizando fertilizante químico (10-18-20), considerando-se uma adubação de base de $300 \mathrm{~kg} \mathrm{ha}^{-1}$ e ureia como adubação de cobertura, na dose $110 \mathrm{~kg} \mathrm{ha}^{-1}$, para produzir sorgo forrageiro. De formar similar, Alves Filho et al. (2003) encontraram valores inferiores ao deste trabalho, cerca de US\$220,60 ha ${ }^{-1}$, utilizando adubação de base com formulado e ureia na adubação de cobertura, para formação de pastagem (azevém). Esta diferença está ligada ao valor do tipo de manejo adotado, o tipo de adubo, bem como, o tipo de solo que está sendo utilizado.

\begin{tabular}{|c|c|c|c|c|}
\hline \multicolumn{2}{|c|}{ Valor do AQC* } & Constituição & Dose recomendada de AQC & Custo do AQC (Caq) \\
\hline SFS & *US\$ 0,24 kg ${ }^{-1}$ & $18 \%$ de $\mathrm{P}_{2} \mathrm{O}_{5}$ & $80 \mathrm{~kg} \mathrm{ha}^{-1} \mathrm{ano}^{-1}$ de $\mathrm{P}_{2} \mathrm{O}_{5}$ & US\$ $105,00 \mathrm{ha}^{-1}$ \\
\hline $\mathrm{KCl}$ & *US\$ 0,38 kg & $58 \%$ de $\mathrm{K}_{2} \mathrm{O}$ & $* * 300 \mathrm{~kg} \mathrm{ha}^{-1}$ de $\mathrm{K}_{2} \mathrm{O}$ & US\$ $195,00 \mathrm{ha}^{-1}$ \\
\hline Ureia & *US\$ 0,40 kg-1 & $44 \%$ de $N$ & $* * 300 \mathrm{~kg} \mathrm{ha}^{-1}$ de $\mathrm{N}$ & US\$ $270,00 \mathrm{ha}^{-1}$ \\
\hline \multicolumn{4}{|c|}{ Total } & US\$ 570,00 ha \\
\hline
\end{tabular}

*preço pesquisado no mercado de atacado; **Dose correspondente a seis cortes na forrageira.

Tabela 5: Valor dos fertilizantes utilizados na adubação química convencional, constituição, doses recomendadas para aplicação e custos com dessa adubação em 24 semanas de cultivo do capim-elefante.

Valor similar ao encontrado neste trabalho foi gasto por Soares et al. (2001) com a aplicação de ureia na produção de aveia preta e azevém, durante o período de inverno, que foi de cerca de US\$278,50 ha ${ }^{-1}$, ao aplicar uma dose de $200 \mathrm{~kg} \mathrm{ha}^{-1}$ de N. Os autores ressaltaram que 
a adubação nitrogenada de cobertura com ureia representou $36 \%$ do custo total da produção. Segundo Neumann et al. (2005), o custo com a adubação foi o responsável por $25 \%$ dos custos totais da produção da forrageiras, enquanto para Alves Filho et al. (2003) este valor chegou a $56 \%$, resultados que reforçam o quanto é dispendioso financeiramente o uso de AQC.

Na Tabela 6 está apresentada a economia com AQC, caso seja usada a fertirrigação com EDP. O EDP gerou uma economia de $100 \%$ do $\mathrm{P}_{2} \mathrm{O}_{5}, 33 \%$ do $\mathrm{K}_{2} \mathrm{O}$, e $93 \%$ do $\mathrm{N}$, ou seja, o valor da Ef foi maior que o Caq apenas para a dose de $\mathrm{P}_{2} \mathrm{O}_{5}$, o que proporcionou uma economia total de US\$ 445,00 $\mathrm{ha}^{-1}$.

\begin{tabular}{l|c}
\hline Dose Fertirrigação (Df) & Economia com fertirrigação (Ef) \\
\hline $97 \mathrm{~kg} \mathrm{ha}^{-1}{\text { de } \mathrm{P}_{2} \mathrm{O}_{5}}$ & US\$ $127,00 \mathrm{ha}^{-1}$ \\
\hline $100 \mathrm{~kg} \mathrm{ha}^{-1}$ de $\mathrm{K}_{2} \mathrm{O}$ & US\$ $65,00 \mathrm{ha}^{-1}$ \\
\hline $280 \mathrm{~kg} \mathrm{ha}^{-1}$ de N & US\$ $253,00 \mathrm{ha}^{-1}$ \\
\hline Total & US\$ 445,00 ha \\
\hline
\end{tabular}

Tabela 6: Dose de N, P e K e economia com adubação química, caso seja substituída pela fertirrigação com EDP, após 24 semanas de aplicação.

$\mathrm{O}$ valore de $\mathrm{P}_{2} \mathrm{O}_{5}$ foi $22 \%$ maior que o aportado ao solo por fertilização convencional. Assim, a quantidade de $\mathrm{P}_{2} \mathrm{O}_{5}$ aplicados via EDP foi 1,2 vezes maior que o indicado por Ribeiro et al. (1999) para manter altas produtividades de forrageiras.

A utilização da fertirrigação com EDP em 24 semanas de aplicação, estabelecida utilizando-se o $\mathrm{Na}$ como elemento químico referência, representou, para o atual ano, uma economia de US\$ 445,00 ha ${ }^{-1}$, equivalente a uma dose de 97, 100 e $280 \mathrm{~kg} \mathrm{ha}^{-1}$ de $\mathrm{P}_{2} \mathrm{O}_{5}, \mathrm{~K}_{2} \mathrm{O}$ e N, respectivamente, e representando uma economia de $78 \%$ do custo total com AQC. Cabe ressaltar que essa economia será maior, se levar em conta que haverá gasto com mão-de-obra na aplicação de AQC.

Com apenas a dose de $\mathrm{P}_{2} \mathrm{O}_{5}$ aplicada via fertirrigação estando maior que as recomendações de $A Q C$, estas não irão refletir em maiores produtividades com EDP, tendo em vista que a dose de $\mathrm{N}$ dita a produtividade e a dose fertirrigada $\left(280 \mathrm{~kg} \mathrm{ha}^{-1}\right)$ foi próxima da dose com AQC (300 kg ha-1), sendo assim, espera-se que a produtividade seja semelhante ou pouco menor. Estudo realizado por Vitor et al. (2009) demonstraram que o aumento na dose nitrogenada até $700 \mathrm{~kg} \mathrm{ha}^{-1} \mathrm{ano}^{-1}$ (24 semanas equivale a $351 \mathrm{~kg} \mathrm{ha}{ }^{-1}$ de $\mathrm{N}$ ), aumentou linearmente a produtividade do capim-elefante.

Para efeito de alimentação animal, com o aumento na produtividade da forrageira, aumentará a disponibilidade desta por unidade de área, o que, em termos práticos, resultará em uma maior pressão de pastejo, ou seja, aumentará a quantidade de animais na mesma área (RESTLE et al., 2002). Isso significa que a produtividade por peso animal e o ganho financeiro, por unidade de área, será maior.

Os custos apresentados abaixo levam em consideração os gastos relativos ao processo de produção e a aplicação da irrigação/fertirrigação (TABELA 7). Os custos com mudas não foram levados em consideração, uma vez que geralmente são conseguidos na própria propriedade, bem como, o custo com energia da irrigação, entendendo que o sistema será implantado todo por diferença de nível, não utilizando energia elétrica. 


\begin{tabular}{|c|c|c|}
\hline Custos & AQC & EDP \\
\hline Custo de produção: & \multicolumn{2}{|c|}{----- US\$ ha ${ }^{-1}$} \\
\hline Mudas & 0,00 & 0,00 \\
\hline${ }^{1}$ Arrendamento da terra & 35,00 & 35,00 \\
\hline \multicolumn{3}{|l|}{ Fertilizantes: } \\
\hline - Ureia & 271,00 & 0,00 \\
\hline - SS & 105,00 & 0,00 \\
\hline$-\mathrm{KCl}$ & 195,00 & 0,00 \\
\hline \multicolumn{3}{|l|}{ Mecanização: } \\
\hline 2- Plantio & 53,00 & 53,00 \\
\hline 1- Aplicação de fertilizante & 53,00 & 0,00 \\
\hline 1- Mão-de-obra & 46,00 & 46,00 \\
\hline Custo total de produção & 758,00 & 134,00 \\
\hline \multicolumn{3}{|l|}{ Custo Irrigação: } \\
\hline 3 - Custo implantação & 680,00 & 680,00 \\
\hline 1. Custo aplicação & 965,00 & 965,00 \\
\hline 4- Custo manutenção & 20,00 & 20,00 \\
\hline 5- Custo água * ITN & 11,00 & 5,00 \\
\hline Custo total de irrigação & 849,00 & 843,00 \\
\hline Custo total & $1.607,00$ & 977,00 \\
\hline
\end{tabular}

Tabela 7: Custos de produção e custo de irrigação (sulcos), por unidade de área receptora de adubação química convencional (AQC) ou efluente do tratamento preliminar de esgoto doméstico (EDP), após 24 semanas de cultivo.

Adaptado por: ${ }^{1}$ - (NEUMANN et al., 2005); ${ }^{2}$-; ${ }^{3}$-(BERNARDO et al., 2008); ${ }^{4}$-(LEITCH, 1983 apud SOARES et al., 2002); ${ }^{5}$ - (DN CBH-VELHAS, no03/2009).

O custo total de produção com aplicação de AQC foi de US\$758,00 ha ${ }^{-1}$, cerca de $82 \%$ maior que a com aplicação de EDP, que foi de US\$ 134,00 ha ${ }^{-1}$. Essa diferença ressalta o quanto foi vantajoso a utilização de EDP, do ponto de vista econômico, estando essa diferença ligada ao custo com fertilizantes e os gastos de mão-de-obra para a sua aplicação. Valores inferiores na produção de forrageiras utilizando AQC foram encontrados por Alves Filho et al. (2003), Neumann et al. (2005) e Soares et al. (2001), que foram, respectivamente, de US\$398,90; 365,00; e 278,50 $\mathrm{ha}^{-1}$.

A diferença no custo de irrigação entre os que receberam AQC e EDP foi irrisória, cerca de 0,6\%, o que ressalta o baixo valor cobrado pela agência regulamentadora pelo metro cúbico de água, US\$ 0,003 $\mathrm{m}^{-3}$. Essa baixa influência do custo da água no custo da irrigação, foi apresentada por Gomes (2005) para irrigação, por sulcos, da cultura do tomateiro.

O custo total da irrigação foi de US\$ 849,00 e 843,00 ha $^{-1}$, nas parcelas submetidas aos tratamentos com AQC e EDP, respectivamente, esses valores foram maiores que o reportado por Gomes (2005), que foi de US\$ $675,80 \mathrm{ha}^{-1}$, utilizando sistema por sulcos simplificado com utilização de mangueiras.

O custo total da produção de capim-elefante utilizando AQC foi, então, de US\$1.607,00 $\mathrm{ha}^{-1}$, enquanto a utilização de EDP gerou um custo total de US\$977,00 ha ${ }^{-1}$, essa diferença foi de US\$ $630,00 \mathrm{ha}^{-1}$, o que representou uma economia $60 \%$ do custo total. 


\section{CONCLUSÕES}

De maneira geral, o EDP se mostrou de excelente qualidade em relação aos seus atributos químicos, podendo suprir a demanda nutricional do capim-elefante. Essa água residuária foi capaz de substituir $100 \%$ de $\mathrm{P}_{2} \mathrm{O}_{5}, 33 \%$ de $\mathrm{K}_{2} \mathrm{O}$, e $93 \%$ de $\mathrm{N}$ para essa cultura, gerando uma economia financeira de US\$ 445,00 ha-1 para adubação; de US\$ 624,00 ha ${ }^{-1}$ no custo de produção; e de US\$ 6,00 ha ${ }^{-1}$ no custo de irrigação, proporcionando uma economia total de US\$ 630,00 ha ${ }^{-1}$ e uma economia hídrica de $58 \%$ do total demandado.

Faz-se importante lembrar que os dados deste trabalho se referem a um período de observações de 6 meses e por se tratar de uma planta de cultivo praticamente permanente, é possível que alguns impactos (principalmente na condutividade elétrica do solo) sejam potencializados ao longo de aplicações constantes do EDP, sendo este mais um argumento a favor do uso cauteloso, respeitando as doses recomendadas dessa técnica para produção forrageira visando a alimentação animal.

\section{REFERÊNCIAS}

ABDEL-AZIZ, R. (2015). Impact of Treated Wastewater Irrigation on Soil Chemical Properties and Crop Productivity. Journal of Water Resources and Arid Environments, v. 4, n. 1, p. 30-36.

ALVES FILHO, D. C.; NEUMANN, M.; RESTLE, J.; SOUZA, A. N. M.; PEIXOTO, L. A. (2003). Características agronômicas produtivas, qualidade e custo de produção de forragem em pastagem de azevém (Lolium multiflorum Lam) fertilizada com dois tipos de adubo. Ciência Rural, v. 33, n. 1, p. 143-149.

AMERICAN PUBLIC HEALTH ASSOCIATION - APHA.(2005). Standard methods for the examination for water and wastewater. Washington, 21a ed., $1220 \mathrm{p}$.

BERNARDO, S.; SOARES, A. A.; MANTOVANI, E. C. (2008). Manual de Irrigação - 8a ed., 625 p.

CBH-VELHAS. DELIBERAÇÃO NORMATIVA DA COMISSÃO DA BACIA HIDROGRÁFICA DO RIO DAS VELHAS, DEN ${ }^{\circ}$ 03/2009 de 20 de março 2009. 2009.

DOORENBOS, J.; KANSSAM, A. H. (1994).Efeito da água no rendimento das culturas. Tradução de: GHEYI, H.R.; SOUZA, A. A.; DAMASCO, F.A.V.; MEDEIROS, J. F. Campina Grande, UFPB, 306 p. (FAO: Irrigação e drenagem, n. 33).

DOORENBOS, J.; PRUITT, W. O. (1997). Guidelines for predicting crop water requirements. Roma, FAO, 179p. (FAO: Irrigation and Drainage Paper, 24).

EMBRAPA, EMPRESA BRASILEIRA DE PESQUISA AGROPECUÁRIA. (2015). Informações sobre a cultivar de capim-elefante BRS Kurumi. Juiz de Fora - MG, 4 p. (Comunicado Técnico, 75)

EMBRAPA, EMPRESA BRASILEIRA DE PESQUISA AGROPECUÁRIA. (1999). Manual de análises químicas de solos, plantas e fertilizantes. Comunicação para Transferência de Tecnologia, Brasília, Brasil, $370 \mathrm{p}$.

FONSECA, A. F.; MELFI, A. J.; MONTES, C. R. (2005). Maize Growth and Changes in Soil Fertility After Irrigation with Treated Sewage Effluent. II. Soil Acidity, Exchangeable Cations, and Sulfur, Boron, and Heavy Metals Availability. Communications in Soil Science and Plant Analysis, v. 36, n. 14, p. 1983-2003.

GOMES, E. P. (2005).Viabilidade de mudanças tecnológicas na irrigação da tomaticultura de mesa. 2005. Universidade Estadual de Campinas, Tese de doutorado, 93p.

HAMILTON, A. J.; STAGNITTI, F.; XIONG, X.; KREIDL, S. L.; BENKE, K. K.; MAHER, P. (2007). Wastewater irrigation: The state of play. Vadose Zone Journal, v. 6, n. 4, p. 823-840. 
KIHILA, J.; MTEI, K. M.; NJAU, K. N. (2014). Wastewater treatment for reuse in urban agriculture; the case of Moshi Municipality, Tanzania. Physics and Chemistry of the Earth, v. 72, p. 104110.

LEAL, R. M. P.; HERPIN, U.; FONSECA, A. F. da; FIRME, L. P.; MONTES, C. R.; MELFI, A. J. (2009). Sodicity and salinity in a Brazilian Oxisol cultivated with sugarcane irrigated with wastewater. Agricultural Water Management, v. 96, n. 2, p. 307-316.

MATOS, A. T. (2014). Tratamento e aproveitamento agrícola de resíduos sólidos. 1ạ ed. Viçosa: Editora UFV.

MATOS, A. T. (2015). Manual de análises de resíduos sólidos e águas residuárias. 1ạ ed. Viçosa: Editora UFV.

MATOS, A. T. (2012). Qualidade do meio físico ambiental: práticas de laboratório. 1a ed. Viçosa: Editora UFV.

MOYO, L. G.; VUSHE, A.; JANUARY, M. A.; MASHAURI, D. A. (2015). Evaluation of suitability of Windhoek's wastewater effluent for re-use in vegetable irrigation: a case study of Gammams effluent. v. 199, p. 109-120.

NEUMANN, M.; RESTLE, J.; FILHO, D. C. A.; MACCARI, M.; PELLEGRINI, L. G.; SOUZA, A. N. M.; PEIXOTO, L. A. O. (2005). Qualidade de forragem e desempenho animal em pastagem de sorgo (Sorghum bicolor), fertilizada com dois tipos de adubo, sob pastejo contínuo. Revista Brasileira de Agrociência, v. 11, n. 2, p. 221-226.

RESTLE, J.; ROSO, C.; AITA, V.; NÖRNBERG, J. L.; BRONDANI, I. L.; CERDÓTES, L.; CARRILHO, C. O. (2002). Produção animal em pastagem com gramíneas de estação quente. Revista Brasileira de Zootecnia, v. 31, n. 3, p. 1491-1500.

RIBEIRO, A. C.; GUIMARÃES, P. T. G.; ALVAREZ V., V. H. (1999). Comissão de Fertilidade do Solo do Estado de Minas Gerais - Recomendações para o uso de corretivos e fertilizantes em Minas Gerais - 5a Aproximação. Viçosa, MG, 359p.

SOARES, A. B.; RESTLE, J.; ROSO, C.; LUPATINI, G. C.; ALVES FILHO, D. C. (2001) Dinâmica, qualidade, produção e custo de produção de forragem da mistura aveia preta e azevém anual adubada com diferentes fontes de nitrogênio. Ciência Rural, v. 31, n. 1, p. 117-122.

SOARES, J. I.; COSTA, R. N. T.; SILVA, L. A. C.; GONDIM, R. S. (2002). Função da resposta da melancia aos níveis de água e adubação nitrogenada, no Vale do Curu, CE. Revista Brasileira de Engenharia Agrícola e Ambiental, v. 6, n. 2, p. 219-224.

THAPLIYAL, A.; VASUDEVAN, P.; DASTIDAR, M. G.; TANDON, M.; SEN, P. K.; MISHRA, S. (2011).Growth of tree saplings of neem (Azadirachtaindica Juss) under fertigation with untreated and treated domestic wastewater. Journal of Scientific and Industrial Research, $v$. 70, n. 8, p. 616-621.

THORNTHWAITE, C. W. (1948).An Approach toward a Rational Classification of Climate. Geographical Review, v. 38, n. 1, p. 55-94.

VITOR, C. M. T.; FONSECA, D. M.; CÓSER, A. C.; MARTINS, C. E.; NASCIMENTO JÚNIOR, D.; RIBEIRO JÚNIOR, J. I. (2009). Produção de matéria seca e valor nutritivo de pastagem de capimelefante sob irrigação e adubação nitrogenada. Revista Brasileira de Zootecnia, v. 38, n. 3, p. 435-442.

VON SPERLING, M. (2014). Introdução à qualidade das águas e ao tratamento de esgotos. Belo Horizonte. 4a ed, v. 1, 472 p. 